\title{
Efficiency Dynamics on Two Coupled Small-World Networks
}

\author{
Jin-Fang Zhang and Zhi-Gang Shao \\ Institute of Modern Physics, Chinese Academy of Sciences, Lanzhou 730000 \\ and Graduate School of the Chinese Academy of Sciences, Beijing 100000 China \\ Lei Yang* \\ Institute of Modern Physics, Chinese Academy of Sciences, Lanzhou 730000 \\ and Department of Physics, Lanzhou University, Lanzhou 730000 China
}

(Received on 6 July, 2010)

\begin{abstract}
We investigate the effect of clusters in complex networks on efficiency dynamics by studying a simple efficiency model in two coupled small-world networks. It is shown that the critical network randomness corresponding to transition from a stagnant phase to a growing one decreases to zero as the connection strength of clusters increases. It is also shown for fixed randomness that the state of clusters transits from a stagnant phase to a growing one as the connection strength of clusters increases. This work can be useful for understanding the critical transition appearing in many dynamic processes on the cluster networks.
\end{abstract}

Keywords: Efficiency dynamics, Small-world networks, Cluster.

\section{INTRODUCTION}

In recent years, complex networks have attracted much attention in various fields [1-3]. In the studies of complex networks, an important issue is to investigate the effect of their complex topological features on dynamic processes taking place upon the networks [4-10]. The topological features, such as degree distribution, clustering coefficient, degreedegree correlation, and so on, are mostly concerned. Lately, it has been determined that many real-world networks show cluster structures [11-13]. Cluster networks are relevant to many social and biological phenomena [14-18]. Cluster networks consist of a number of clusters, where nodes within each group are densely connected, while the linkage among the groups is sparse. Among the many outstanding problems concerning cluster networks, the propagation of information, such as rumor, news, or facts [19], and the propagation of mass or energy [20] are of great interest. However, there are few works about the influences of various degrees of cluster structure upon dynamics [21, 22].

In the past, a simple model which describes the dynamics of efficiencies of competing agents [23] was developed on a small-world network and on scale-free networks with the tunable degree exponents $[24,25]$. In this model communications among agents lead to the increase of efficiencies of underachievers, and the efficiency of each agent can increase or decrease irrespective of other agents. The model has been found useful in modelling the dynamics of a variety of systems, including force fluctuations in granular systems such as bead packs, river networks, voting systems, wealth distributions, size distributions of fish schools, inelastic collisions in granular gases, the generalized Hammersley process, particle systems in one dimension, and various generalized mass transport models [26-33].

In this paper, based on the recently addressed problem of "cluster" in complex networks, we studied a simple efficiency model in the two coupled small-world networks. The present work can be useful for understanding the critical tran-

${ }^{*}$ Electronic address: lyang@impcas.ac.cn sition appearing in many dynamic processes on the cluster networks.

\section{MODEL AND METHOD}

\subsection{Two coupled small-world networks}

First, two separated small-world networks are constructed. An one-dimensional small-world network can be established as follows [34]: The starting point is a ring with $N$ nodes, in which each node is symmetrically connected with its $2 K$ nearest neighbors. Then, for every node each link connected to a clockwise neighbor is rewired to a randomly chosen node with probability $x$, and preserved with probability $1-x$. Self-connections and multiple connections are prohibited, and realizations for which the small-world network becomes disconnected are discarded. As advanced above, the parameter $x$ measures the randomness of the resulting small-world networks. Being independent of the value of network randomness $x$, the average number of links per site $\langle k\rangle$ is always $2 K$. And then, $M$ links are randomly connected between two small-world networks. If $M=0$, the two networks are separated clusters. The system size is $2 N$.

\subsection{Efficiency model}

The evolution of the efficiencies is the one used by S.Y. Huang et al. [24] and Z.-G. Shao et al. [25], which may mimic the dynamics of efficiencies of competing agents such as airlines, travel, agencies, insurance companies and so on. Our efficiency model on the two coupled small-world networks can be described as follows: Each vertex $i$ in the network represents an agent, which is characterized by a nonnegative integer number $h_{i}(t)$. This value stands for its efficient level. The higher $h_{i}$ is, the more advanced (efficiently speaking) the agent is. We assume that the interaction makes the efficiencies of underachievers try to equal to the efficiencies of better performing agents. The interactions between the agents are expressed by the networks. The calculated 
results for present model are independent of the initial conditions [23-25]. For simplicity, we set the efficiency of each agent as $h_{i}(t)=0$ in the initial conditions. Monte Carlo (MC) simulations have been used to study the evolution of the efficiencies of $N$ agents in the small-world network. At each MC step, an agent $i$ is selected at random and its efficient level is updating according to rules $[24,25]$ :

(I) $h_{i}(t) \rightarrow \max \left[h_{i}(t), h_{j}(t)\right]$ with probability $1 /(1+p+q)$, where the agent $j$ is one of the agents which are linked to the agent $i$. This move is due to the fact that each agent tries to equal its efficiency to that of a better performing agent in order to stay competitive.

(II) $h_{i}(t) \rightarrow h_{i}(t)+1$ with probability $p /(1+p+q)$. This incorporates the fact that each agent can increase its efficiency due to innovations, irrespective of other agents.

(III) $h_{i}(t) \rightarrow h_{i}(t)-1$ with probability $q /(1+p+q)$. This corresponds to the fact that each agent can lose its efficiency due to unforeseen problems such as labor strikes. Note, however, that since $h_{i}(t) \geq 0$, this move can occur only when $h_{i}(t) \geq 1$.

The evolution of efficiency continues step by step. After each MC step the 'time' is increased by $1 / N$, so after 1 time step on average all agents in the network have made an update. Because we mainly investigate the effect of the clusters in complex networks on efficiency dynamics, the parameters $p$ and $q$ are held fixed for the two clusters, namely $p=1.5$ and $q=7.5$, which are used in Ref. 24 .

\section{RESULTS AND DISCUSSION}

Extensive numerical simulations were done to investigate the dynamics of efficiency on the two coupled small-world networks. In the simulations, we take the size of each cluster as $N=10^{4}$. To reduce the effect of fluctuation the calculated results are averaged over both 10 different network realizations and 10 independent runs for each network realization.

Firstly, we set $M=0$ to study efficiency dynamics on a single small-world network. As shown by S.-Y. Huang et al. [24] for fixed $p$ and $q$, there exists a critical phase transition from a stagnant phase of efficiency to a growing phase of efficiency at a critical $x_{c}$. To characterize this transition, we calculate the growth rate $v$ of the average efficiency $\langle h(t)\rangle$ per agent in the long-time limit,

$$
v \equiv \frac{d\langle h(t)\rangle}{d t},
$$

where

$$
\langle h(t)\rangle=\frac{1}{N} \sum_{i=1}^{N} h_{i}(t) .
$$

This transition can be also characterized by the efficiency fluctuation $w$ of the system, which corresponds to the nonuniform degree of efficiencies in the system. The efficiency fluctuation $w(t)$ is defined as

$$
w^{2}(t)=\frac{1}{N} \sum_{i=1}^{N}\left(h_{i}(t)-\langle h(t)\rangle\right)^{2} .
$$

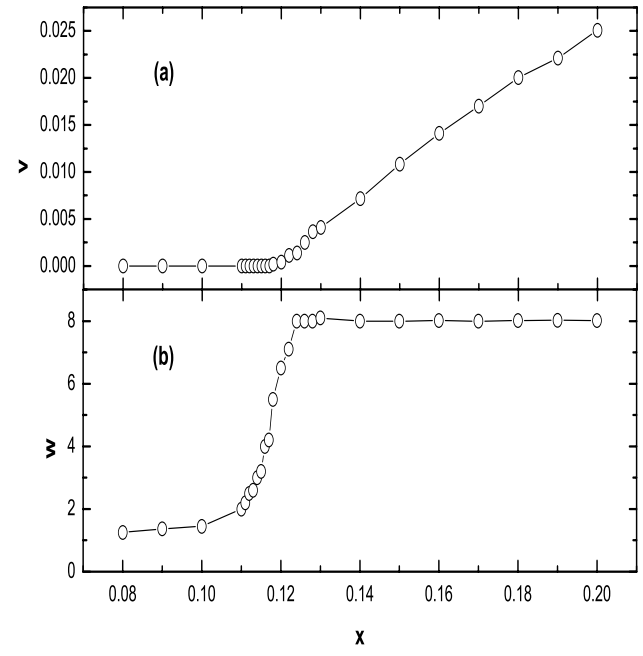

FIG. 1: (a) The asymptotic growth rate $v$ of the average efficiency and (b) the asymptotic efficiency fluctuation $w$ as functions of network randomness $x$.

In the long-time limit, the efficiency fluctuation $w(t)$ tends to a constant $w=\langle w(t \rightarrow \infty)\rangle$.

Figure 1(a) shows the growth rate $v$ of the average efficiency as a function of $x$. It can be seen from this figure that there exists a transition at a certain value $x_{c}$. As $x>x_{c}, v$ increases rapidly with $x$; As $x<x_{c}$, growth rate $v$ is equal to zero. As $x \approx x_{c}$, growth rate $v$ transits from zero to a finite value, which corresponds to the transition of the system from a stagnant phase to a growing one. Figure 1(b) shows the asymptotic value $w$ as a function of $x$. From Fig. 1(b) we can see that fluctuation $w$ also shows a transition behavior similar to that of growth rate $v$. From the Fig. 1(b), we obtain that $x_{c}=0.12$.

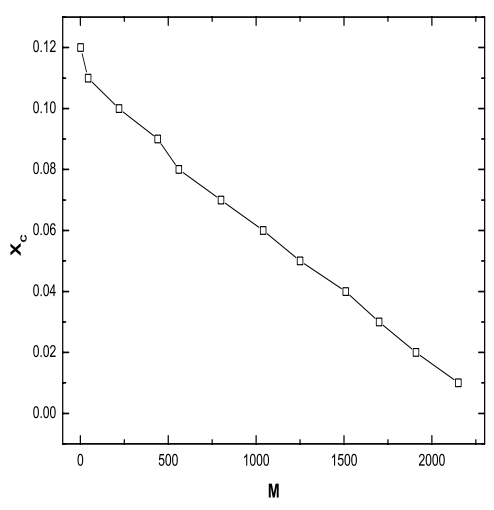

FIG. 2: The critical network randomness $x_{c}$ as a function of $M$.

Secondly, we study the efficiency dynamics on the networks $A$ and $B$, which are characterized by the same rewiring probabilities, namely $x_{A}=x_{B}$. Figure 2 shows critical network randomness $x_{c}$ as a function of $M$. As long as $M$ increases, $x_{c}$ decreases and we have that $x_{c}=0$ for $M \geq m$. For 
$M=0$, and $x_{A}=x_{B}<x_{C}=0.12$, the states of two clusters are both the stagnant phase. When $x_{c}$ decreases to a value smaller than $x_{A}$, the states of the two clusters transit from the stagnant phases to the growing one. For $M$ is larger than a certain value $m$, the transition disappears, which indicates that the state of the two clusters is always the growing phase independent of network randomness $x$.

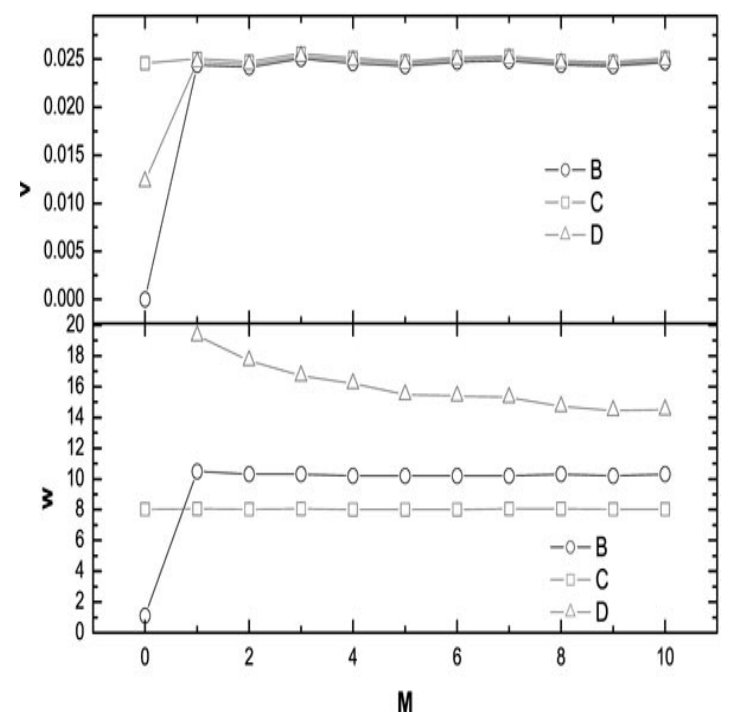

FIG. 3: (color online) (a) The growth rate $v$ of the average efficiency and (b) the asymptotic value $w$ in the clusters A, B, and global network as functions of $M$.

Finally, let us study the efficiency dynamics on the networks when $x_{A}=0.2$ and $x_{B}=0.01$ are fixed. When $M=0$, the state of cluster $A$ is a growing phase, and the state of cluster B is a stagnant phase. Figure 3 shows the growth rate $v$ of the average efficiency and the asymptotic value $w$ in the clusters $\mathrm{A}, \mathrm{B}$, and global network as functions of $M$, where the global network consists of the clusters $A$ and $B$. As shown in Fig. 3 we can see that the states of two clusters are both growing phases as $M \geq 1$. From the curve of the asymptotic values $w$ of the global network as a function of $M$, we obtain that the critical point is given by $M_{c}=1$. We can still find that only one link between two clusters can change the state of efficiency dynamics. It is a guidance to develop economics of two different regions. For example, cooperations between a wealthy region and a poor region or between two poor regions are significantly positive to the development of economics.

In the following, we try to understand the critical behavior by analyzing the dynamic properties of the present model. Firstly, we write down the evolution equation for the average efficiency $\langle h(t)\rangle$ per agent. The contributions to the time evolution of $\langle h(t)\rangle$ come from three parts: increase due to learning from its linked agents, increase due to innovation, and decrease due to unforeseen problems. Thus, the growth rate $v$ of the average efficiency can be expressed as [23-25]

$$
v(t) \equiv \frac{d\langle h(t)\rangle}{d t}=\frac{r w(t)+p-q s(t)}{1+p+q},
$$

where $r$ is a proportional factor concerning with the $M$ and

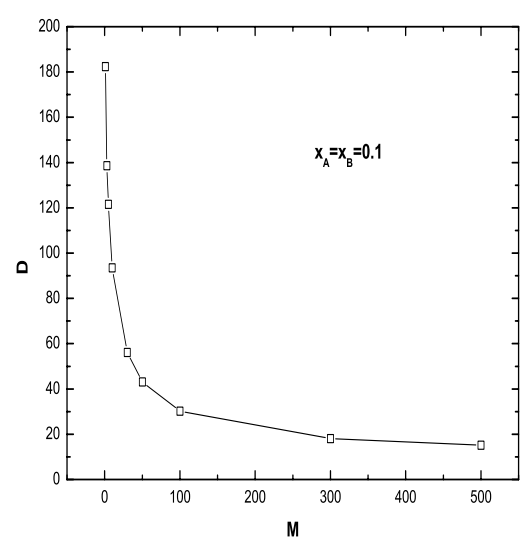

FIG. 4: The distance of global network $D$ as a function of $M$ with $x_{A}=x_{B}=0.1$.

$x$, and $s(t)$ is the probability that an agent has a nonzero efficiency. The first term on the right-hand side of the above equation indicates the increase in efficiency per agent due to the fact that each agent tries to equal its efficiency to that of a better performing agent, which is proportional to the nonuniform degree $w$ of efficiencies among agents. The second term represents the increase in efficiency per agent due to the innovation of each agent. The last term quantifies the loss in efficiency per agent due to some unforeseen problems, taking into account the fact that the reduction can take place from an agent only if the agent has a nonzero efficiency.

Secondly, based on the above Eqs. (4), we analyze the two different situations. For the situation of efficiency dynamics on the networks with the same parameters. Figure 4 shows the distance of global network $D$ as a function of $M$ with $x_{A}=x_{B}=0.1 . D$ is the average shortest path length which is a measure of the typical separation between two nodes in the global network, namely

$$
D=\frac{2}{N(N-1)} \sum_{i j} d_{i j}
$$

where $d_{i j}$ is the optimal path length from node $i$ to node $j$.

From Fig. 4 one can see that $D$ decreases when $M$ increases. The growth rate $v$ increases because the first term $r w(t)$ increases. Therefore, the $x_{c}$ will decrease as the $M$ increases. For the situation of efficiency dynamics on the networks with different parameters, $x_{A}=0.2$ and $x_{B}=0.01$. Because there is a growing phase in the cluster $\mathrm{A}$ and one link between the two clusters, the term $r w(t)$ related to the cluster B is proportional to the same term related to cluster A. Therefore the state of the cluster $\mathrm{B}$ is a growing phase.

\section{CONCLUSION}

To investigate the effect of the clusters on efficiency dynamics in complex networks, we studied a simple efficiency model in two coupled small-world networks. As the connection strength of clusters increases, the state of clusters transits from a stagnant phase to a growing one, and the critical network randomness $x_{c}$ decreases to zero. We hope that the 
present work will also be useful for understanding the critical transition appearing in many dynamic processes on complex networks with clusters, optimizing or controlling dynamic processes on social or biological networks.

\section{Acknowledgments}

This work was supported by the 100 Person Project of the Chinese Academy of Sciences, the China National Natural
Science Foundation with Grant No. 10775157.
[1] R. Albert, A.-L. Barabási, Rev. Mod. Phys. 74, 47 (2002).

[2] S. N. Dorogovtsev, J. F. F. Mendes, Adv. Phys. 51, 1079 (2002).

[3] M. E. J. Newman, SIAM Rev. 45, 167 (2003).

[4] R. Pastor-Satorras and A. Vespignani, Phys. Rev. Lett. 86, 3200 (2001)

[5] M. Barthélemy, A. Barrat, R. Pastor-Satorras, and A. Vespignani, Phys. Rev. Lett. 92, 178701 (2004).

[6] T. Nishikawa, A. E. Motter, Y.-C. Lai, and F. C. Hoppensteadt, Phys. Rev. Lett. 91, 014101 (2003).

[7] V. M. Eguíluz and K. Klemm, Phys. Rev. Lett. 89, 108701 (2002).

[8] M. Chavez, D.-U. Hwang, A. Amann, H. G. E. Hentschel, and S. Boccaletti, Phys. Rev. Lett. 94, 218701 (2005).

[9] D.-H. Kim, B. J. Kim, and H. Jeong, Phys. Rev. Lett. 94, 025501 (2005).

[10] A. Castro e Silva, J. K. L. da Silva, and J. F. F. Mendes, Phys. Rev. E 70, 066140 (2004)

[11] M. E. J. Newman, Phys. Rev. E 64, 016131 (2001).

[12] M. Girvan and M. E. J. Newman, Proc. Natl. Acad. Sci. U.S.A. 99, 7821 (2002).

[13] G. Palla, I. Derényi, I. Farkas, and T. Vicsek, Nature 435, 814 (2005).

[14] J. R. Banavar, A. Maritan, and A. Rinaldo, Nature 390, 130 (1999).

[15] J. K. L. da Silva, G. J. M. Garcia, and L. Barbosa, Phys. life Revs. 3, 229 (2006).

[16] J. K. L. da Silva, L. A. Barbosa, Braz. J. Phys. 39, 699 (2009).

[17] J. Camacho, and A. Arenas, Nature 435, E4 (2005).

[18] L. A. Barbosa, A. Castro e Silva, and J. K. L. da Silva, Phys. Rev. E 73, 041903 (2006).
[19] V. Schwammle, M. C. Gonzáles, A. A. Moreira, J. S. Andrade Jr., and H. J. Herrmann, Phys. Rev. E 75, 066108 (2007).

[20] L. A. Barbosa, and J. K. L. da Silva, Europhys. Lett. 90, 30009 (2010).

[21] G. Yan, Z.-Q. Fu, J. Ren, and W.-X. Wang, Phys. Rev. E 75, 016108 (2007)

[22] L. Huang, K. Park, and Y.-C. Lai, Phys. Rev. E 73, R035103 (2006).

[23] S. N. Majumdar, P. L. Krapivsky, Phys. Rev. E 63, 045101 (2001).

[24] S.-Y. Huang, X.-W. Zou, Z.-J. Tan, Z.-G. Shao, Z.-Z. Jin, Phys. Rev. E 68, 016107 (2003).

[25] Z.-G. Shao, J.-P. Sang, Z.-J. Tan, X.-W. Zou, and Z.-Z. Jin, Eur. Phys. J. B 48, 587 (2005).

[26] T. Halpin-Healy, Y.-C. Zhang, Phys. Rep. 254, 215 (1995)

[27] H. Hinrichsen, R. Livi, D. Mukamel, A. Politi, Phys. Rev. Lett. 79, 2710 (1997).

[28] S. N. Majumdar, S. Krishnamurthy, M. Barma, Phys. Rev. E 61, 6337 (2000)

[29] S. Ispolatov, P. L. Krapivsky, and S. Redner, Eur. Phys. J. B 2, 267 (1998).

[30] S. N. Coppersmith, C.-h. Liu, S. Majumdar, O. Narayan, and T. A. Witten, Phys. Rev. E 53, 4673 (1996).

[31] A. Maritan, A. Rinaldo, R. Rigon, A. Giacometti, and I. R. Iturbe, Phys. Rev. E 53, 1510 (1996).

[32] M. Cieplak, A. Giacometti, A. Maritan, A. Rinaldo, I. R. Iturbe, and J. R. Banavar, J. Stat. Phys. 91, 1 (1998).

[33] E. Bonabeau and L. Dagorn, Phys. Rev. E 51, R5220 (1995).

[34] D. J. Watts and S. H. Strogatz, Nature 393, 440 (1998). 